significantly associated with RAP ( $\mathrm{p}=0.55)$ or parasite infection, as concurrent infection was present in only 2 patients $(p=0.24)$.

Conclusion From this study, low grade inflammation, manifest by increased IELs, may be associated with RAP and also parasitic infection. $H$ pylori is not associated with parasite infection. However, as eosinophilia was not significantly associated with the condition further investigation is required to elucidate the potential involvement of innate immunity, including mast cells. Furthermore, there is no association between $H$ pylori infection and RAP. Funded by EU CONTENT Project (INCO-CT-2006-032136), CONICYT/ BM (RUE \#29) and Fondecyt \#1100654 (Chile).

Competing interests None declared.

\section{PTU-133 READY TO GO AND LET GO: PERSPECTIVES ON TRANSITION AND TRANSFER FROM PAEDIATRIC TO ADULT HEALTH CARE: A PAIRED PILOT SURVEY OF ADOLESCENT IBD PATIENTS AND THEIR PARENTS}

doi:10.1136/gutjnl-2012-302514c.133

N Rao, ${ }^{*}$ D Ashok, A Azaz, S Sebastian. Hull \& East Yorkshire NHS Trust, Hull, UK

Introduction National surveys report that transition care in inflammatory Bowel Disease is still not well developed. Although the general principles guiding transition of adolescents with chronic illnesses from paediatric to adult health care have been established, there are no studies to assess the transfer needs and concerns of adolescents with IBD and their carers. We aimed to gauge the perspectives of adolescents with IBD and their parents to determine their issues, concerns and expectations.

Methods A cross sectional survey of IBD patients starting transition process in a dedicated transition clinic and their parents was undertaken using a semi-structured questionnaire designed for self completion. Along with demographic and clinical information, respondents were asked to rate on a scale of $1-5$ (using Likert scale anchored by 1 -least important and 5 -very important and essential) their responses on their perceived importance on the aspects of preparation, self management skills, concerns regarding transfer and value of support services.

Results 20 patients (12 Crohn's and 8 Ulcerative Colitis) and their parents completed the survey independently. There was concordance in the responses of patients and their parents in rating highly the need for information, education and co-ordination of transfer process (Abstract PTU-133 table 1). The knowledge, empathy and accessibility were identified as key attributes for the transferring adult team (Abstract PTU-133 table 2). The highest rated concern for both adolescents and their parents was the perceived differences in performing tests such as endoscopy (mean score of $4.55 \pm 0.17$ and $4.65 \pm 0.23$ respectively). The adolescents favoured to attend appointments without parents before transfer (mean score

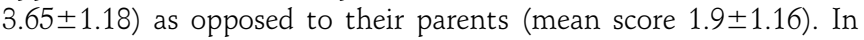
addition parents were concerned whether they will become less involved in care following transfer (mean score 4.15. The adolescents' favoured younger age of transition and transfer when compared to their parents. While dedicated adolescent services were described as beneficial by patients and their parents, only $50 \%$ of patients preferred to have joint appointments.

Conclusion This first pilot study demonstrates significant concerns about transition process needing addressing among adolescent IBD patients and their parents. Patients and parents differ in their rating of independence and self advocacy needed at the time of transfer. Tailoring transition to individual patient and parent needs without dedicated transition care teams may be challenging.

Competing interests None declared.
PTU-134 WHAT DO YOUNG PEOPLE AND PARENTS WANT FROM AN INFLAMMATORY BOWEL DISEASE (IBD) SERVICE?

doi:10.1136/gutjnl-2012-302514c.134

${ }^{1} \mathrm{R}$ Little, ${ }^{* 2} \mathrm{C}$ Imrie, ${ }^{3} \mathrm{~A}$ Derby, ${ }^{3} \mathrm{P}$ Gillespie, ${ }^{1} \mathrm{G}$ R Caddy, ${ }^{1} \mathrm{~T} \mathrm{C}$ Tham. ${ }^{1}$ Gastroenterology, Ulster Hospital, Dundonald, Belfast, Belfast, UK; ${ }^{2}$ Altnagelvin Hospital, Derry/Londonderry, UK; ${ }^{3}$ Northern Ireland branch, Crohns and Colitis UK, Belfast, UK

Introduction At present, there are guidelines from the US and Europe regarding the formation of transition clinics for adolescents with IBD. This includes a UK Inflammatory Bowel Disease (IBD) Standards guidance on optimal service provision for paediatric and adolescent care. However most of these guidelines come from intuitive reasoning and opinion, as there is a lack of data on what constitutes an ideal service for young patients with IBD. The aim of this study was to develop a comprehensive knowledge and understanding of the key service requirements of young people with IBD as well as their parents.

Methods Paediatric and adolescent patients age 6-18 years, were identified from databases in two teaching hospitals and from the membership of the N Ireland branch of Crohn's and Colitis UK, which is a patient support group. Anonymous questionnaires were sent to these patients and their parents separately. The questionnaires asked about their perceived quality of care, clinic care, general comments, input from specialists, support and information, plus any suggestions.

Results 105 questionnaires were sent and 51 responded (49\%); of these 21 were from patients and 30 from their parents. Over $84 \%$ were happy with the quality of care they are receiving. Reasons patients and parents were reluctant to attend clinics included: blood tests, nurse specialist or doctor not available, lack of car parking. $90 \%$ preferred to see the attending (Consultant) rather than a fellow. Nurse specialist, dietetics, specialist IBD surgeon, psychologist, skin/ eye specialist input was thought to be beneficial by $95 \%, 81 \%, 71 \%$, $59 \%$, and $45 \%$ respectively. The following support service and information were considered important: immediate contact with healthcare personnel for disease flare, support groups for young adults, insurance and financial advice, knowledge about IBD developments and research, email service, surgical input regarding stomas.

Conclusion The majority of young patients with IBD and their parents are satisfied with the care they are receiving. Support from specialist services such as nurse specialist, dietitians, specialist IBD surgeons, psychologist, plus rapid access to services when the disease flares were thought to be important by the patients and their parents. Knowledge of what these patients and their parents want will help to design an optimal IBD service.

Competing interests R Little: None Declared, C Imrie Conflict with: Mead Johnson, Falk Pharma, Nutricia, Warner Chilcott, SHS, Norgine, Wyeth, A Derby: None declared, P Gillespie: None declared, G Caddy: None declared, T Tham Speaker bureau with: Warner Chilcott, Shire, Conflict with: Abbott, MSD.

\section{PTU-135 INCIDENCE OF HP INFECTION IN CHILDREN AND THE ASSESSMENT OF HISTOLOGICAL STAINING PRACTICE}

doi:10.1136/gutjnl-2012-302514c.135

${ }^{1} \mathrm{Z}$ M Mead, ${ }^{1} \mathrm{~B}$ Vadgama, ${ }^{2} \mathrm{R}$ Mead, ${ }^{*}{ }^{1} \mathrm{D}$ Fowler, ${ }^{1} \mathrm{~S}$ Holden. ${ }^{1}$ Paediatric Histopathology, University Hospital Southampton NHS Trust, Southampton, UK; ${ }^{2}$ Gastroenterology, Hampshire Hospitals NHS Trust, Basingstoke, UK

Introduction The exact incidence of Helicobacter pylori (HP) in childhood is unknown. There is a causal link between HP and development of peptic ulcers, gastric adenocarcinoma and lymphoma. However there is little evidence and guidance on the clinical management of HP in children. The North American Society 
for Paediatric Gastroenterology and Nutrition report there is no compelling evidence to treat children colonised with HP without histological evidence of gastritis. This reflects the current pathophysiological understanding of cancer development, where inflammation secondary to infection is believed to be the driver of dysplasia and cancer. In our tertiary referral centre current practice stains all paediatric gastric biopsies with Haematoxylin and Eosin stain $(\mathrm{H}+\mathrm{E})$ as well as Warthin Starry (WS) stain, specifically for HP-like organisms. The aim of this study was to investigate the incidence of HP infection in children undergoing endoscopy. We also examined the routine use of special staining on all gastric biopsies for $\mathrm{HP}$ and whether other histological features on $\mathrm{H}+\mathrm{E}$ examination could act as reliable markers of infection allowing selective special staining.

Methods We reviewed all gastric biopsies submitted between January 8 and September 11, in patients born after 1989. All reports were loaded onto a database and searched for key words with "helico", "HLO" and "H Pylori". Each report was then examined for evidence of HP, acute inflammation and lymphoid aggregates

Results 1102 cases were examined and 23/1102 were positive for HP, $2 \%$ of all cases. There was acute inflammation in $17 / 23$ cases. In the six with no acute inflammation, two were suspected of having HP (one previously treated) and one had only a single biopsy from the body, not the antrum. 146/1102 (13\%) cases had acute inflammation. Acute inflammation as a marker for HP has a sensitivity of $74 \%$, specificity of $88 \%$, PPV of $11 \%$ and NPV of $99 \%$. Lymphoid aggregates and acute inflammation as markers of infection further increased HP detection.

Conclusion This study shows our current practice involves special staining of all biopsies for low prevalence disease (2\%). However $\mathrm{H}+\mathrm{E}$ examination alone has low sensitivity for picking up HP in cases of low density infection. Acute inflammation as a marker for HP infection could aid selection of cases for special staining. The NPV of $99 \%$ suggests that very few cases would be missed. Selective staining of cases for HP would also save around £3000/ year (excluding pathologist time). The disadvantage that a small number of children colonised with HP, without gastritis may be missed is of unclear significance. Further research is required in order to develop an integrated clinical and pathological approach into the investigation, diagnosis and treatment of HP in children.

Competing interests None declared.

\section{Pathology}

\section{PTU-136 DOES TOTAL NUMBER AND POSITIVE LYMPH NODE RATIO HAVE AN IMPACT ON OUTCOME FOLLOWING SURGICAL RESECTION FOR HILAR CHOLANGIOCARCINOMA?}

doi:10.1136/gutjnl-2012-302514c.136

A Hakeem, ${ }^{*}$ G Marangoni, S Chapman, R Young, A Nair, E Hidalgo, G Toogood, J P A Lodge, K R Prasad. Department of HPB Surgery, St James's University Hospital NHS Trust, Leeds, UK

Introduction Lymph node status is an important predictor of survival following resection for hilar cholangiocarcinoma (HCCA). Controversies still exist regarding the extent of lymphadenectomy and whether an extended lymph node dissection improves outcome. This study aims to evaluate the prognostic value of the total number of nodes removed and positive lymphnode ratio (LNR) on overall and disease-free survival in patients undergoing resection for HCCA.

Methods From 1994 to 2010, 84 HCCA were resected at our Institution. Seventy-eight patients with available data were included in our analysis. Overall survival (OS) and disease-free survival (DFS) were calculated and stratified according to the number of lymph nodes excised and positive LNR at different cut-off levels.

Results An average of 13.8 lymph nodes were removed. 45 patients $(57.7 \%)$ had a positive lymph node status, with a mean of 3.2 involved nodes per patient. 1, 5 and 10-year OS for $\mathrm{N}+$ status was $60 \%, 10 \%$ and $10 \%$, while N- OS was $82 \%, 41 \%$ and $41 \%(p=0.000)$ Similarly, 1, 5 and 10 -year DFS was worse in the $\mathrm{N}+$ group $(71 \%$, $45 \%$ and $42 \%)$ compared to $\mathrm{N}-(91 \%, 65 \%$ and $60 \%)(\mathrm{p}=0.045)$ There was no difference in 1, 5 and 10 -year OS $(70 \%, 23 \%, 20 \%$ vs $70 \%, 23 \%$ and $20 \%, \mathrm{p}=0.690)$ and DFS $(78 \%, 48 \%$ and $48 \%$ vs $82 \%$, $58 \%$ and $58 \%, p=0.305)$ when $<10$ nodes were removed $(n=39)$ compared to $\geq 10$ nodes $(n=36)$. There was no difference in 1,5 and 10 -year OS (63\%, $9 \%$ and $9 \%$ vs $60 \%, 10 \%$ and $10 \%, p=0.562)$ and DFS $(78 \%, 40 \%$ and $40 \%$ vs $65 \%, 46 \%$ and $40 \%, p=0.795)$ when LNR $<0.25(n=22)$ was compared to LNR $>0.25 \quad(n=23)$. No difference was found when a cut-off of 15 total excised lymphnodes and LNR of 0.50 was used.

Conclusion The overall number of lymphnodes excised and positive LNR did not correlate with overall and DFS in resected HCCA. Larger, prospective studies are necessary to confirm these results.

Competing interests None declared.

\section{PTU-137 DOES A PROMPT FROM THE HISTOLOGY REPORT TO PERFORM SEROLOGY TO EXCLUDE COELIAC DISEASE MEAN THIS IS DONE?}

doi:10.1136/gutjnl-2012-302514c.137

${ }^{1} \mathrm{E}$ Byrne, ${ }^{*}{ }^{1} \mathrm{~K}$ Lloyd, ${ }^{2} \mathrm{~S}$ Nayagam, ${ }^{2} \mathrm{H}$ Williams, ${ }^{1} \mathrm{M}$ Walker. ${ }^{1}$ Histopathology, Imperial NHS Healthcare Trust, London, UK; ${ }^{2}$ Gastroenterology, Imperial NHS Healthcare Trust, London, UK

Introduction Lymphocytic duodenosis (LD) is a histological diagnosis comprising normal duodenal architecture, but an intraepithelial lymphocyte (IELs) count $>25 / 100$ enterocytes. A diagnosis of coeliac disease (CD) can be made in up to $16 \%$ of cases where $\mathrm{LD}$ is seen. ${ }^{1} \mathrm{CD}$ serology (antibodies to tissue transglutaminase) should be performed in all cases of LD. The aim of this study was to identify the rate of $\mathrm{CD}$ serological investigations following a diagnosis of $\mathrm{LD}$. Methods All duodenal biopsies taken at St Marys Hospital, London between March and August 2011 were evaluated. The histological reports for all duodenal biopsies coded as abnormal were reviewed. Serology results were checked in all cases where $\mathrm{LD}$ was diagnosed. Results 280 duodenal biopsies were performed of which 24 met the criteria for a diagnosis of $\mathrm{LD}(8.6 \%)$. The indication for biopsy was anaemia in $12 / 24(50 \%)$, dyspepsia in $4 / 24(16.7 \%)$, abdominal pain in $3 / 24(12.5 \%)$, dysphagia in $1 / 24(4.2 \%)$ and an indication was not indicated in $1 / 24$ (4.2\%). The numbers of lymphocytes per 100 enterocytes varied from 26 to 62 . Serological testing for CD was carried out prior to or following the diagnosis of $\mathrm{LD}$ in $12 / 24(50 \%)$. In all cases where serology was performed the result was negative. Conclusion Opportunities to diagnose CD are being missed in 50\% of cases. These figures are similar to an audit carried out in the same department in 2009 which showed that serological testing for CD was being carried out in $56 \%$ of cases where LD was diagnosed. Diagnosis and treatment of CD has been shown to reduce morbidity and mortality ${ }^{2} 3$ and reduce the cost of medical care. ${ }^{4}$ Therefore it is important that opportunities to diagnose CD are taken and followup on the diagnosis of $\mathrm{LD}$ with serological testing by clinicians is imperative.

Competing interests None declared.

\section{REFERENCES}

1. Aziz I, Evans $K E$, Hopper $A D$, et al. A prospective study into the aetiology of lymphocytic duodenosis. Aliment Pharmacol Ther 2010;32:1392-7.

2. Rubio-Tapia A, Kyle RA, Kaplan EL, et al. Increased prevalence and mortality in undiagnosed coeliac disease. Gastroenterology 2009;137:88-93. 